\title{
Terlipressina como Novo Recurso Terapêutico no Choque Séptico*
}

\section{Terlipressin as a New Therapeutic Agent in Septic Shock}

\author{
Valter Nilton Felix ${ }^{1}$
}

\section{RESUMO}

JUSTIFICATIVA e OBJETIVOS: A terlipressina tem sido inserida em protocolos de suporte hemodinâmico da sepse, como recurso em casos de choque refratário, o que motiva análise crítica a respeito do assunto. CONTEÚDO: Foram revistas para a análise terapias hemodinâmicas com objetivos finais bem delineados e novas recomendações para reanimação volêmica, uso de vasopressores e agentes inotrópicos em adultos e crianças sépticos.

CONCLUSÕES: A terlipressina tem sido considerada nova alternativa nos cuidados intensivos da sepse, embora ainda controversa.

Unitermos: choque séptico, terapia vasopressora, Terlipressina.

\section{SUMMARY}

BACKGROUND AND OBJECTIVES: The hemodynamic support of sepsis is now formulated trying to insert terlipressin as salvage drug in catecholamine resistant shock, justifying a broad critical analysis.

CONTENTS: The analysis included hemodynamic the-

1. Professor Livre-Docente do Departamento de Gastroenterologia do HCFMUSP; Coordenador Científico da Unidade de Terapia Intensiva do Departamento de Gastroenterologia do HCFMUSP

Presidente da Comissão Científica da SOPATI; Coordenador de Eventos da AMIB

*Recebido do Departamento de Gastroenterologia do HCFMUSP, São Paulo, SP

Apresentado em 13 de março de 2006

Aceito para publicação em 10 de maio de 2006

Endereço para correspondência:

Dr. Valter Nilton Felix

Rua Frei Caneca, 1407 Cj. 221 - Cerqueira César

01307-909 São Paulo, SP

Tel.: (11) 3287-7456 Fax (11) 3283-2715

Email: v.felix@terra.com.br

(C)Associação de Medicina Intensiva Brasileira, 2006 rapies with defined specific goals and new recommendations for fluid resuscitation, vasopressor therapy, and inotropic therapy of septic in adult and pediatric patients.

CONCLUSIONS: Terlipressin appears as a new but controversial alternative for vasopressor therapy in sepsis.

Key Words: septic shock, Terlipressin, vasopressor therapy

\section{INTRODUÇÃO}

O choque séptico tem caráter distributivo e cursa com vasodilatação. No geral, os pacientes apresentam o índice cardíaco elevado e o índice de resistência vascular sistêmica reduzido; desta forma, é muito relevante o suporte cardiovascular efetivo, buscando atingir a pressão arterial média capaz de garantir perfusão tecidual, transporte e consumo de oxigênio compatíveis com preservação funcional de órgãos e sistemas.

Os vasopressores, portanto, tem grande valor terapêutico, destacando-se a noradrenalina e a dopamina ${ }^{1}$.

Entretanto, existem casos em que tais substâncias, mesmo quando administradas em doses elevadas, são ineficazes, com redução progressiva da resposta vascular com o passar do tempo, no chamado choque refratário ${ }^{2}$.

Atribui-se o fenômeno, modernamente, a formação excessiva de óxido nítrico associado a ativação de canais de potássio sensíveis a ATP e bloqueio dos canais de entrada celular de cálcio ${ }^{3}$.

A dramaticidade de tais situações justifica a busca de alternativas e a vasopressina tem sido estudada em vários ensaios clínicos, mostrando ser um fármaco capaz de promover vasoconstrição, via receptores $\mathrm{V} 1$, acoplados à fosfolipase $\mathrm{C}$, aumentando a concentração de cálcio intracelular, ação não bloqueada pela sepse, podendo assim reverter a hipotensão resistente a catecolaminas daqueles pacientes ${ }^{4,5}$.

A terlipressina é análogo sintético da vasopressina, 
mantendo farmacodinâmica semelhante, mas farmacocinética diferente, pois enquanto a vida média da vasopressina é de seis minutos, a da terlipressina chega a seis horas, portanto, com maior possibilidade de ser empregada com sucesso no choque séptico refratário, como já sugerido em ensaio clínico recente, que, no entanto, não avaliou o transporte e o consumo de oxigênio, nem os efeitos específicos sobre os rins ou o fígado.

Este estudo despertou interesse investigativo clínico e experimental no sentido de determinar possível inserção do fármaco em guias protocolados de atendimento do choque séptico.

\section{O AVANÇO DAS INVESTIGAÇÕES}

Surgiram trabalhos que identificaram redução do índice cardíaco, em função da diminuição da freqüência cardíaca, com comprometimento de transporte e consumo de oxigênio (avaliação por calorimetria) e alteração da função hepática, contrapondo-se a melhora da função renal, com utilização da terlipressina em infusão contínua de 10 a $40 \mu \mathrm{g} / \mathrm{kg} / \mathrm{min}^{7}$ ou em bolus de $1 \mathrm{mg}$, repetida depois de 20 minutos, caso não haja resposta pressórica ${ }^{8}$.

No primeiro estudo, os níveis de lactato sérico não se alteraram, chegando a decrescer no segundo, especulando-se que a melhora hemodinâmica possa ter implementado a distribuição tecidual de oxigênio.

Quanto à melhora da função renal, tem sentido, com base em análises da resposta da síndrome hepato-renal ao fármaco ${ }^{9}$.

Nenhum dos dois trabalhos mostrou alteração da função cardíaca, recomendando-se o uso da telipressina no estado hiperdinâmico refratário, com atenção especial ao índice cardíaco (o que deveria limitar a dose a ser empregada), com pressão ocluída de artéria pulmonar (POAP) entre 12 e $15 \mathrm{mmHg}$, embora recentemente já se estabeleça tal parâmetro entre 8 e $12 \mathrm{mmHg}^{10}$.

O emprego da terlipressina em situação clínica semeIhante, também sem grupo controle (e dificilmente será possível tê-lo em estudo clínico) e com pequeno número de casos, foi efetuado em 14 crianças de 7 dias a 17 anos de idade, comparando-se o período de 12 horas antes do uso do fármaco com o mesmo tempo depois de sua administração, que começava com 7 $\mu \mathrm{g} / \mathrm{kg}$ duas vezes por dia e, dependendo da resposta da pressão arterial e dos efeitos colaterais concorrentes, podia chegar a $20 \mu \mathrm{g} / \mathrm{kg}$ a cada 6 horas $^{11}$.

Seis crianças sobreviveram, com aumento estatistica- mente significativo da pressão arterial média observada 10 minutos após a aplicação da terlipressina. $A$ freqüência cardíaca, o índice de oxigenação e o lactato sérico diminuíram, o débito urinário e a $\mathrm{PaO}_{2}$ aumentaram à luz de análises estatísticas.

A meia-vida longa da terlipressina permite que sua aplicação em bolus provoque efeito em 10 a 20 minutos, mas, sobretudo, que se espere que sua ação perdure por mais 5 horas, o que já havia sido observado previamente $^{12}$ e foi considerado na realização daquele estudo, que optou pela aplicação periódica, e não contínua, do fármaco.

Outros trabalhos já haviam sugerido benefícios hemodinâmicos do uso de terlipressina no choque séptico refratário em crianças ${ }^{13,14}$, mas agora também é acrescido que estímulo dos receptores V2 dos vasos pulmonares possa promover vasodilatação e reduzir o shunt pulmonar. Isto também seria devido à redução das doses dos demais vasoconstritores ${ }^{15}$.

Seus resultados, portanto, contestaram o aumento de resistência vascular pulmonar, tido como possível efeito adverso da terlipressina, utilizada na endotoxemia experimental ${ }^{7,16}$.

Também nas crianças demonstrou-se aumento de diurese, atribuída a redução da vasoconstrição arteriolar aferente, com a redistribuição de fluxo sistêmico.

A terlipressina tem maior afinidade aos receptores vasculares V1 e menor aos V2, em relação à vasopressina ${ }^{6}$ e não aumenta a atividade fibrinolítica tal qual a vasopressina $^{7}$, o que a privilegia no tratamento do choque séptico, em que seriam de valia seus efeitos de aumento da pós-carga do ventrículo esquerdo.

Fisiologicamente a concentração sérica de vasopressina endógena aumenta de forma significativa na hemorragia e na sepse, mas adultos com choque séptico hipotensivo e vasodilatado teriam deficiência relativa de vasopressina ${ }^{17}$.

Isto foi demonstrado, com efeito, ocorrer em cerca de um terço dos casos de choque séptico tardio ${ }^{18}$.

Tais pacientes, portadores de síndrome de deficiência hormonal, por depleção neuro-hipofisária ou déficit de liberação, costumam ser hipersensível à vasopressina na sepse, em que dose de 0,01 a 0,07 unidades $/ \mathrm{min}$ costuma alterar substancialmente a pressão arterial, enquanto infusão de 0,2 a 2 unidades/minuto raramente atua naquele sentido em portadores de sangramento de varizes do esôfago ${ }^{19,20}$.

O uso da terlipressina na hemorragia digestiva e na síndrome hepato-renal transcorre com raros efeitos colaterais ${ }^{9,21-23}$, mas no choque séptico são preocupantes 
os relatos de diminuição do índice cardíaco, a par da almejada elevação da resistência vascular periférica e de que muitas vezes o fato de o débito cardíaco estar aumentado pode amenizar sua redução com o emprego do fármaco ${ }^{24}$.

A reflexão ainda traz preocupações sobre a possibilidade de manutenção dos fenômenos inflamatórios com a redução do fluxo esplâncnico, sem contar que a instabilidade do choque séptico pode dificultar a correta titulação das doses subseqüentes, mesmo após primeira resposta positiva.

De qualquer forma, o enfoque de tratamento de síndrome de deficiência hormonal (quanto à vasopressina) na sepse é bastante atraente e parece justificar estudos que produzam maior embasamento, mesmo porque o "choque vasodilatado" de difícil controle de outras causas, por exemplo, o hemorrágico tardio e o que segue circulação extracorpórea prolongada ${ }^{25}$, disputariam lugar na mesma esteira.

Alguns dos importantes estudos sobre os principais efeitos da terlipressina no choque séptico constam da tabela 1.

\begin{tabular}{|c|c|c|c|c|c|}
\hline Autores & Casuística & Dose & PAM & Diurese & $\begin{array}{c}\text { Índice } \\
\text { Cardíaco }\end{array}$ \\
\hline $\begin{array}{l}\text { O'Brien A } \\
\text { e col. }{ }^{6}\end{array}$ & 8 & $1 \mathrm{a} 2 \mathrm{mg}$ & $>$ & $>$ & $<$ \\
\hline $\begin{array}{l}\text { Leone M } \\
\text { e col. }{ }^{8}\end{array}$ & 17 & $1 \mathrm{mg}$ & $>$ & $>$ & $<$ \\
\hline $\begin{array}{l}\text { Matok, I } \\
\text { e col. }{ }^{11}\end{array}$ & 14 & 7 a $20 \mu \mathrm{g} / \mathrm{kg}$ & $>$ & $>$ & $<$ \\
\hline $\begin{array}{l}\text { Perner A } \\
\text { e col. }{ }^{29}\end{array}$ & 1 & $0,5 \mathrm{mg}$ & $>$ & $<$ & $<$ \\
\hline $\begin{array}{l}\text { Morelli } \\
\text { e col. }{ }^{30}\end{array}$ & 15 & $1 \mathrm{mg}$ & $>$ & $>$ & $<$ \\
\hline
\end{tabular}

PAM - pressão arterial média

\section{ENFOQUE ESPECÍFICO DO FLUXO ESPLÂNCNICO}

Dados importantes têm sido obtidos em estudos experimentais. Após a indução de endotoxemia em ratos, tratados de início com reanimação volêmica, para depois receberem pequena dose de terlipressina, tiveram recuperação da pressão arterial sistêmica estatisticamente significativa, sem diminuição do índice de fluxo aórtico (tomado como referência de índice cardíaco) e com manutenção de adequado fluxo micro-circulatório ileal; ao contrário, os animais tratados diretamente com terlipressina nem sequer obtiveram elevação da pressão arterial ${ }^{26}$, o que mostrou que não tem sentido pensar no fármaco como substituto de outros im- portantes passos na terapêutica global da sepse, mas também que a redução reflexa do índice cardíaco pode não ser óbice definitivo ao seu emprego.

É possível que o acerto volêmico inadequado explique relatos sumários de aumento do gradiente mucoso-arterial de $\mathrm{CO}_{2}$, à tonometria gástrica ou do L-lactato no lume retal, em pacientes sépticos tratados com infusão de terlipressina ou vasopressina ou dose única de terlipressina ${ }^{27-29}$.

De fato, aquele efeito nocivo é contestado em estudo clínico que envolveu uso de tonometria gástrica e técnica de fluxometria laser-doppler ${ }^{30}$.

Foram estudados 15 pacientes com choque séptico, recebendo altas doses de norepinefrina para sustentar a pressão arterial média de 50 a $55 \mathrm{mmHg}$. Foram introduzidos probes de tonometria e de laser-doppler na câmara gástrica e os pacientes receberam bolus, por via venosa, de $1 \mathrm{mg}$ de terlipressina como terapia de resgate.

Houve, em 30 minutos, aumento estatisticamente significativo da pressão arterial média e da perfusão da mucosa gástrica, com diminuição do débito cardíaco, todos sustentados por 24 horas.

A relação da perfusão da mucosa gástrica com o consumo sistêmico de oxigênio também aumentou, enquanto o gradiente entre a pressão parcial mucosa-arterial de $\mathrm{CO}_{2}$ tendeu a diminuir, com estatística significativa depois de 8 horas $(p<0,05)$.

O débito urinário aumentou progressivamente, com incremento estatisticamente significativo da depuração de creatinina e na redução da necessidade de norepinefrina.

Chama a atenção que todos os pacientes preencheram os critérios de classificação de choque séptico ${ }^{31}$, com APACHE II médio de 27, foram submetidos a reanimação volêmica até atingir POAP de $14 \mathrm{mmHg}$, estavam sob ventilação mecânica, sedados com midazolam ou sufentanil, e recebiam 0,6 $\mu \mathrm{g} / \mathrm{hg} / \mathrm{min}$ ou mais de norepinefrina, por pelo menos 36 horas, com pressão arterial média de $51 \pm 1,5 \mathrm{mmHg}$.

O detalhe importante do protocolo é que, enquanto a norepinefrina foi sendo reduzida, com o surgimento de parâmetros arteriais mais convenientes, foi mantida infusão de hidroxietilstarch a $6 \%$, iniciada com $70 \mathrm{~mL} / \mathrm{h}$, para manter a POAP constante durante o estudo, assim como foi administrada, de forma contínua a dobutamina na dose de $5 \mu \mathrm{g} / \mathrm{kg} / \mathrm{min}$.

Não se discute melhora de sobrevida com o emprego da terlipressina, mesmo porque se trata de pequena amostra, mas atribui-se a seu uso, inegável melhora distributiva de fluxo, embora não se tenha grupo controle. 


\section{CONCLUSÃO}

Não se pode negar interesse ao assunto, mesmo porque o fármaco foi incluído no Surviving Sepsis Campaign Guidelines ${ }^{10}$, recomendando-se infusão contínua (de vasopressina) de 0,01-0,04 unidades/min no choque refratário, com grau $\mathrm{E}$ de recomendação, o que fez aumentar o interesse de desenvolvimento de novos protocolos clínicos, meta atual do Serviço, enfocando não a vasopressina, mas a terlipressina.

\section{REFERÊNCIAS}

01. Reinhart K, Sakka SG, Meier-Hellmann - Haemodynamic management of a patient with septic shock. Eur J Anaesthesiol, 2000;17:6-17.

02. Astiz ME, Rackow EC - Septic shock. Lancet, 1998;351:(9114):15011505.

03. Takakura K, Taniguchi T, Muramatsu I et al - Modification of alpha1-adrenoceptors by peroxynitrite as a possible mechanism of systemic hypotension in sepsis. Crit Care Med, 2002;30:894-849.

04. Dunser M, Mayr AJ, Ulmer $\mathrm{H}$ et al - The effects of vasopressin on systemic hemodynamics in catecholamine-resistant septic and postcardiotomy shock: a retrospective analysis. Anesth Analg, 2001;93:7-13.

05. Patel BM, Chittock DR, Russell JA et al - Beneficial effects of shortterm vasopressin infusion during severe septic shock. Anesthesiology, 2002;96:576-582.

06. O'Brien A, Clapp L, Singer M - Terlipressin for norepinephrine resistant septic shock. Lancet, 2002;359:1209-1210.

07. Westphal M, Stubbe H, Sielenkamper AW et al - Terlipressin dose response in healthy and endotoxemic sheep: impact on cardiopulmonary performance and global oxygen transport. Intensive Care Med, 2003;29:301-308.

08. Leone M, Albanese J, Delmas A et al - Terlipressin in catecholamineresistant septic shock patients. Shock, 2004;22:314-349.

09. Felix VN - Síndrome hépato-renal. J Bras Gastroenterol, 2005;5:144159.

10. Dellinger RP, Carlet JM, Masur $\mathrm{H}$ et al - Surviving Sepsis Campaign guidelines for management of severe sepsis and septic shock. Intensive Care Med, 2004;30:536-555.

11. Matok, I, Vard, A, Efrati $O$ et al - Terlipressin as rescue therapy for intractable hypotension due to septic shock in children. Shock, 2005;23:305310.

12. Mutlu GM, Factor $P$ - Role of vasopressin in the management of septic shock. Intensive Care Med, 2004;30:1276-1291.
13. Matok I, Leibovitch L, Vardi A et al - Terlipressin as rescue therapy for intractable hypotension during neonatal septic shock. Pediatr Crit Care Med, 2004;5:116-118.

14. Rodriguez-Nunez A, Fernandez-Sanmartin M, Martinon-Torres $F$ et al Terlipressin for catecholamine-resistant septic shock in children. Intensive Care Med, 2004;30:477-480.

15. Thrush DN, Downs JB, Smith RA - Is epinephrine contraindicated during cardiopulmonary resuscitation? Circulation, 1997;96:2709-2714.

16. Scharte M, Meyer J, Van Aken $\mathrm{H}$ et al - Hemodynamic effects of terlipressin (a synthetic analog of vasopressin) in healthy and endotoxemic sheep. Crit Care Med, 2001;29:1756-1760.

17. Delmas A, Leone M, Rousseau S et al - Clinical review: Vasopressin and terlipressin in septic shock patients. Crit Care, 2005;9:212-222.

18. Sharshar T, Blanchard $\mathrm{A}$, Paillard $\mathrm{M}$ et al - Circulating vasopressin levels in septic shock. Crit Care Med, 2003;31:1752-1758.

19. Landry DW, Levin HR, Gallant EM et al - Vasopressin deficiency contributes to the vasodilatation of septic shock. Circulation, 1997;95:11221125.

20. Tsuneyoshi I, Boyle WA - Vasopressin: new uses for an old drug. Contemp Crit Care, 2003;1:1-11

21. Ioannou GN, Doust J, Rockey DC - Systematic review: terlipressin in acute oesophageal variceal haemorrhage. Aliment Pharmacol Ther, 2003;17:53-64.

22. Solanki P, Chawla A, Garg R et al - Beneficial effects of terlipressin in hepatorenal syndrome: a prospective, randomized placebo-controlled clinical trial. J Gastroenterol Hepatol, 2003;18:152-156.

23. Felix VN - Varizes hemorrágicas do esôfago e o emprego da terlipressina. J Bras Gastroenterol, 2002;2:135-138.

24. Peters MJ, Booth R, Petros AJ - Terlipressin bolus induces systemic vasoconstriction in septic shock. Pediatr Crit Care Med, 2004;5:112-115.

25. Morales DL, Garrido MJ, Madigan JD et al - A double-blind randomized trial: prophylactic vasopressin reduces hypotension after cardiopulmonary bypass. Ann Thorac Surg, 2003;75:926-930.

26. Asfar P, Pierrot $\mathrm{M}$, Veal $\mathrm{N}$ et al - Low-dose terlipressin improves systemic and splanchnic hemodynamics in fluid-challenged endotoxic rats. Crit Care Med, 2003;31:215-220.

27. Auzinger GM, O'Callaghan PG, Harry RA et al - Terlipressin in the treatment of catecholamine resistant septic shock. Crit Care, 2002;6:61.

28. Klinzing $\mathrm{S}$, Schiergens $\mathrm{V}$, Reinhart $\mathrm{K}$ et al - Could vasopressin be an alternative for vasopressor therapy in sepsis? Eur J Anaesthesiol, 2002;19:169-170.

29. Perner A, Jorgensen VL, Waldau T - Terlipressin increased the concentration of L-lactate in the rectal lumen in a patient with septic shock. Acta Anaesthesiol Scand, 2004;48:1054-1057.

30. Morelli A, Rocco M, Conti G et al - Effects of terlipressin on systemic and regional haemodinamics in catecholamine-treated hyperkinetic septic shock. Intensive Care Med, 2004;30:597-604.

31. Bone RC, Balk RA, Cerra FB et al - Definitions for sepsis and organ failure and guidelines for the use of innovative therapies in sepsis. Chest, 1992;101:1644-1655. 